\title{
TIMING AND FREQUENCY SYNCHRONIZATION FOR OFDM BASED COOPERATIVE SYSTEMS
}

\author{
Qinfei Huang ${ }^{* 1,2}$, Mounir Ghogho ${ }^{2}$, Jibo Wei ${ }^{1}$ and Philippe Ciblat ${ }^{3}$ \\ ${ }^{1}$ School of Electronic Science \& Engineering, \\ National University of Defense Technology, P. R. China \\ ${ }^{2}$ School of Electronic \& Electrical Engineering, University of Leeds, UK, \\ ${ }^{3}$ Département Comélec, ENST, Paris, France \\ \{eenqh,m.ghogho\}@leeds.ac.uk,wjbhw@nudt.edu.cn,philippe.ciblat@enst.fr
}

\begin{abstract}
In this paper, we investigate the timing and carrier frequency offset (CFO) synchronization problem in decode and forward cooperative systems operating over frequency selective channels. A training sequence which consists of one OFDM block having a tile structure in the frequency domain is proposed to perform synchronization. Timing offsets are estimated using correlation-type algorithms. And since some subcarriers are nulled in the proposed tile structure, CFOs are readily estimated using subspace-based methods. By judiciously designing the size of the tile, these algorithms are shown to have better performance, in terms of synchronization errors and bit error rate, than the computationally demanding SAGE algorithm.
\end{abstract}

\section{INTRODUCTION}

OFDM based cooperative schemes have recently been proposed to combat timing errors (see e.g. [1] and [2].) Indeed, with a cyclic prefix (CP) insertion, OFDM is robust to limited timing errors. However, without compensating for the timing offsets, each OFDM block needs to employ an unnecessarily long CP to mitigate the interblock/multinode interference [3]. This can significantly reduce the data throughput, especially when the expected timing errors are large. Timing synchronization for all the relay nodes is therefore desirable to overcome this problem. Moreover, OFDM systems are very sensitive to carrier-frequency offsets. Therefore, accurate timing and frequency synchronization is key for the deployment of efficient OFDM-based cooperative systems.

To avoid the multidimensional search required by maximumlikelihood (ML) synchronization, a time division multiplexing (TDM) training based synchronization algorithm was proposed in [4]. However, although the resulting algorithms are computationally attractive, the overhead is extremely high since long guard time intervals may be required to avoid overlap of different relay node's signals, especially in mobile networks where the assignment of relay nodes can be highly dynamic. A solution to obtain a good tradeoff between computational complexity and overhead is to multiplex different relay node's training signals in the frequency domain. The synchronization problem then becomes similar to that in uplink OFDMA systems. Thus, synchronization algorithms for uplink OFDMA (see e.g. [5,6] and references therein) may in principle be applied to our problem. However, most of these algorithms need to perform a complicated iterative search to estimate the timing and frequency offsets. Further, iterative-type algorithms are sensitive to initialization. Finally and more importantly, in uplink OFDM, the received signals are processed by the base station which can afford running complex algorithms, whereas in cooperative systems, the receiver may be a mobile unit for which the computational complexity and power consumption are critical issues.

\footnotetext{
*Supported by China Scholarship Council
}

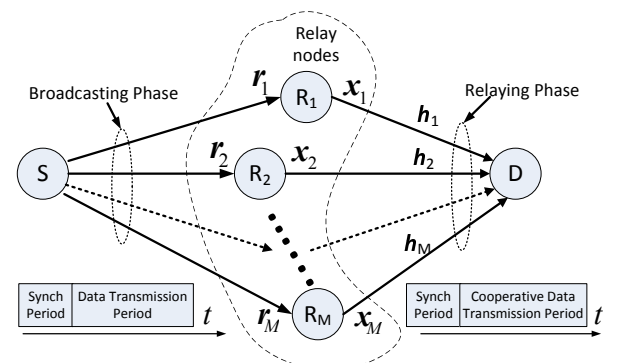

Fig. 1. Cooperative system structure

In this paper, we show that synchronization algorithms with low computational complexity and good performance can be achieved with a single OFDM training block having a tile structure in the frequency domain. Timing offsets are estimated using correlation-type algorithms. The CFOs are estimated using null subcarriers in the tile structure and an ESPRIT-type algorithm. By judiciously designing the size of the tile, these low-complexity algorithms are shown to have better performance, in terms of synchronization errors and bit error rate, than the computationally demanding SAGE algorithm. Unlike [7] which only considers CFO estimation for the interleaved OFDMA uplink, both timing and frequency estimation are investigated in this paper.

\section{COOPERATIVE SYSTEM MODEL}

\subsection{System description}

We consider a decode and forward cooperative system with one source node, one destination node and $M$ relay nodes as shown in Figure 1. Training sequences are used for synchronization.

In the broadcasting phase, the source node broadcasts a training sequence followed by data blocks to the relay nodes. Each relay node operates independently. In the relaying phase, during the synchronization period, the $M$ relay nodes transmit training sequences to the destination node. A multiple parameter estimation task is performed at the destination node. As discussed in [5], accurate timing and frequency compensation cannot be accomplished at the destination node, as the correction of one relay node's offsets would misalign the other relay nodes. To overcome this problem, the destination node may feed back the estimated offsets to the relay nodes. Then, each node can adjust its timing and frequency parameters, so that the data blocks can be synchronized at the destination node. Asynchronous data detection can also be carried out but its complexity may be too high for mobile nodes. It is also worth pointing out that asynchronism may provide delay diversity for flat fading channels but it may have little value in the case of frequency-selective channels. To assess the merits of our synchronization algorithm in terms of bit error rate, we consider in our simulation setup the scenario 


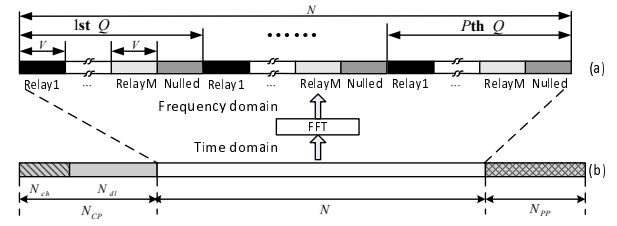

Fig. 2. Training structure illustration

where relay nodes are synchronized using feedback from the destination node and data transmission from the relay nodes is performed using cooperative space-time block coding.

The synchronization problem in the broadcasting phase is similar to that of a downlink OFDMA, for which single user OFDM synchronization techniques can be applied. Hence, we focus on the relaying phase, which is more challenging than the broadcasting phase.

\subsection{Receive signal model}

The received version of the training signal at the destination node can be written as

$$
d(n)=\sum_{i=1}^{M} e^{j 2 \pi \omega_{i} n / N} \sum_{l=0}^{L-1} h_{i}(l) x_{i}\left(n-l-\tau_{i}\right)+v(n)
$$

where $x_{i}(n), \boldsymbol{h}_{i}=\left[h_{i}(0) \cdots h_{i}(L-1)\right]^{T}, \tau_{i}$ and $\omega_{i}$ are respectively the training sequence, the channel impulse response (CIR), the integer part of the normalized (to sampling period) delay and the normalized (to the inverse of the symbol period) CFO associated with the $i$ th relay node, and $v(n)$ is an AWGN with variance $\sigma_{v}^{2}$. Since OFDM modulation is used, the fractional part of the propagation delay is not explicitly included in the above signal model because it can be incorporated into the channel frequency response.

The training sequence for each node consists of a useful part, a cyclic prefix (CP) and cyclic postfix (PP). As in [7], the CP is composed of two parts, $N_{c h}$ and $N_{d l}$. The $N_{c h}$ part of the CP is dedicated to accommodating the channel delay spreads, and the $N_{d l}$ part of the CP and the PP, whose length is denoted by $N_{p p}$, are inserted to accommodate the different propagation delays among relay nodes. It is worth pointing out that the proposed training sequences introduce a lower overhead than TDM training sequences adopted in [4], since long guard time intervals are required by each TDM training sequence. The structure of the proposed training sequence is shown in Figure 2.b. In this paper, we assume that the normalized CFO is less than half of the subcarrier spacing $\left(\left|\omega_{i}\right|<0.5\right)$ and the maximum differential propagation delay $\max \left\{\left|\tau_{i}-\tau_{j}\right|\right\}$ is smaller than $\min \left\{N_{d l}, N_{p p}\right\}$.

The $\mathrm{CP}$ is removed and the first $N$ samples are collected in vector $\boldsymbol{d}=[d(0) \cdots d(N-1)]^{T}$. Letting $\boldsymbol{\Gamma}\left(\omega_{i}\right)=\operatorname{diag}\left\{1, e^{j 2 \pi \omega_{i} / N}\right.$, $\left.\cdots, e^{j 2 \pi \omega_{i}(N-1) / N}\right\} ;\left[\mathbf{X}_{\tau_{i}}\right]_{m, n}=x_{i}\left(m-n-\tau_{i}\right)$, where $m=$ $0, \cdots, N-1$ and $n=0, \cdots, L-1$, and $\boldsymbol{v}=[v(0) \cdots v(N-1)]^{T}$, vector $\boldsymbol{d}$ can, using Eq (1), be expressed as

$$
\boldsymbol{d}=\mathbf{A}_{\boldsymbol{\omega}, \tau} \boldsymbol{h}+\boldsymbol{v}
$$

where $\mathbf{A}_{\boldsymbol{\omega}, \boldsymbol{\tau}}=\left[\boldsymbol{\Gamma}\left(\omega_{1}\right) \mathbf{X}_{\tau_{1}}, \cdots, \boldsymbol{\Gamma}\left(\omega_{M}\right) \mathbf{X}_{\tau_{M}}\right] ; \boldsymbol{h}=\left[\boldsymbol{h}_{1}^{T} \cdots \boldsymbol{h}_{M}^{T}\right]^{T}$; $\boldsymbol{\omega}=\left[\omega_{1}, \cdots, \omega_{M}\right]^{T} ; \boldsymbol{\tau}=\left[\tau_{1}, \cdots, \tau_{M}\right]^{T}$.

The joint ML estimators of the timing and CFO parameters can be readily expressed as

$$
(\hat{\boldsymbol{\tau}}, \hat{\boldsymbol{\omega}})=\arg \max _{\boldsymbol{\tau}, \boldsymbol{\omega}}\left\{\boldsymbol{d}^{H} \mathbf{A}_{\boldsymbol{\omega}, \boldsymbol{\tau}}\left(\mathbf{A}_{\boldsymbol{\omega}, \boldsymbol{\tau}}^{H} \mathbf{A}_{\boldsymbol{\omega}, \boldsymbol{\tau}}\right)^{-1} \mathbf{A}_{\boldsymbol{\omega}, \boldsymbol{\tau}}^{H} \boldsymbol{d}\right\}
$$

The maximization in (3) requires a search over the $2 M$ dimensional domain spanned by $(\boldsymbol{\omega}, \boldsymbol{\tau})$, which may be not tractable in practice. Unlike [4] which uses a TDM training sequence to avoid the multidimensional search, we adopt a frequency-domain multiplexing for our training design in order to obtain low overhead.

\section{TIMING AND CFO ESTIMATION}

\subsection{Training sequence structure description}

The useful part of the training sequence (i.e. excluding CP and PP) for each relay node consists of one OFDM block of DFT size $N$. The total number of activated subcarriers is distributed among the relay nodes as follows. As depicted in Figure 2.a, the $N$ subcarriers are divided into $P$ groups and each group contains $Q$ subcarriers. A tile subchannel is composed by $V$ adjacent subchannels of each group. Parameter $V$ will be referred to as tile size. The number of subcarriers allocated to all the relay nodes in each group is set to be smaller than $Q$, i.e. $M V<Q$, so that null subcarriers, to be used for CFO estimation, can be inserted. The tile subchannel assigned to the $i$ th relay node is composed of subcarriers with index set $\mathcal{M}_{i}=\left\{v+p Q+\kappa_{i} V\right\}_{v=0}^{V-1}$, where $\kappa_{i}$ is an integer in the interval $[0, Q / V-1]$ and $0 \leq p \leq P-1$. Using the above training structure, the received signal can, after removing the $\mathrm{CP}$, be re-expressed as

$$
d(n)=\sum_{i=1}^{M} d^{(i)}(n)+v(n), n=0, \cdots, N-1
$$

where $d^{(i)}(n)=\sum_{v=0}^{V-1} d^{(i, v)}(n)$ and

$$
\begin{array}{r}
d^{(i, v)}(n)=e^{j \phi_{i}} e^{j \theta_{i, v}\left(n-\tau_{i}\right)} \sum_{p=0}^{P-1} X_{i}\left(\eta_{i, v, p}\right) H_{i}\left(\eta_{i, v, p}\right) \\
e^{j 2 \pi p\left(n-\tau_{i}\right) / P}
\end{array}
$$

where $\phi_{i}=2 \pi \omega_{i} \tau_{i} / N ; \theta_{i, v}=2 \pi\left(\omega_{i}+v+\kappa_{i} V\right) / N ; \eta_{i, v, p}=$ $v+p Q+\kappa_{i} V$; and $X_{i}(k)$ and $H_{i}(k)$ are the training sequence and the channel frequency response at the $k$ th subcarriers of the $i$ th relay node, respectively.

\subsection{Timing estimation algorithm}

In this section, we present a Weighted Slide Cross-correlation Timing Estimator (WSCTE), which can estimate each relay node timing parameter readily and accurately by exploring the cross correlation between the received signal and each relay node's training sequence.

Let $Z_{i, k}\left(\tau, \omega_{i}\right) \triangleq \sum_{n=0}^{N-1} d^{(i)}(n-\tau) x_{k}^{*}(n)$. Using eq. (5), we get

$$
Z_{i, k}\left(\tau, \omega_{i}\right)= \begin{cases}0 & i \neq k, \omega_{i}=0 ; \\ Q \sum_{v_{1}=v_{2}=0}^{V-1} Y_{i, i}(\tau, 0) & i=k, \omega_{i}=0 \\ \sum_{v_{1}=0}^{V-1} \sum_{v_{2}=0}^{V-1} \epsilon_{i, k}\left(\omega_{i}\right) Y_{i, k}\left(\tau, \omega_{i}\right) & \omega_{i} \neq 0\end{cases}
$$

where $\epsilon_{i, k}\left(\omega_{i}\right) \triangleq \frac{1-e^{j 2 \pi \omega_{i}}}{1-e^{j 2 \pi\left(\rho_{i, v_{1}}-\rho_{k, v_{2}}+\omega_{i}\right) / Q}}, \rho_{i, v}=v+\kappa_{i} V$ and

$$
\begin{array}{r}
Y_{i, k}\left(\tau, \omega_{i}\right) \triangleq e^{j \phi_{i}} \sum_{n=0}^{P-1} e^{-j \theta_{i, v_{1}} \tau} e^{j 2 \pi\left(\rho_{i, v_{1}}-\rho_{k, v_{2}}+\omega_{i}\right) n / N} \\
g_{i, v_{1}}(n-\tau) f_{k, v_{2}}^{*}(n)
\end{array}
$$

where $f_{i, v}(n) \triangleq \sum_{p=0}^{P-1} X_{i}\left(\eta_{i, v, p}\right) e^{j 2 \pi n p / P}$ and $g_{i, v}(n) \triangleq$ $\sum_{p=0}^{P-1} X_{i}\left(\eta_{i, v, p}\right) H_{i}\left(\eta_{i, v, p}\right) e^{j 2 \pi n p / P}$.

It can be deduced from the first and second terms in the RHS of eq. (6) that the received signal has good correlation properties. However, in the presence of CFO, the above results do not hold anymore. Nevertheless, since the normalized CFO is assumed to be less than half of the subcarriers spacing, it can be verified that the effect of CFO can be neglected as shown in Figure 3. Exploiting multipath diversity, the proposed WSCTE algorithm is given by

$$
\hat{\tau}_{i}=\arg \max _{\tau_{i}} \sum_{l=0}^{L-1} \alpha_{l}\left|\sum_{n=0}^{N-1} d\left(n+\tau_{i}+l\right) x_{i}^{*}(n)\right|
$$



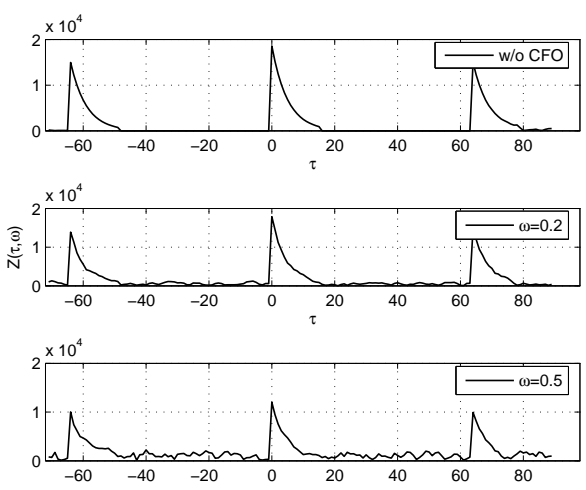

Fig. 3. $Z_{i, k}\left(\tau, \omega_{i}\right)$ as a function of $\omega_{i}$ and $\tau, i=k, N=512$, $L=16, V=3, Q=8$

where $\left\{\alpha_{l}\right\}_{l=0}^{L-1}$ are weighting coefficients. One natural choice of these coefficients is the power distribution of the channel taps, i.e. $\alpha_{l}=\mathrm{E}\left\{\left|h_{i}(l)\right|^{2}\right\}$.

\subsection{CFO estimation algorithm}

Once the timing offset estimation is carried out, we collect samples of the received signal as follows. The starting point of this set of samples is determined by the estimated timing parameter of the relay node whose signal arrives first at the destination. Let $\{\tilde{d}(n)\}_{n=0}^{N-1}$ denote the collected samples. From eq. (4), we get

$$
\begin{array}{r}
\tilde{d}(n+\mu P)=\sum_{i=1}^{M} \sum_{v=0}^{V-1} e^{j \theta_{i, v} \mu P} \tilde{d}^{(i, v)}(n)+v(n+\mu P) \\
n=0, \cdots, P-1, \mu=0, \cdots, Q-1
\end{array}
$$

We then arrange the $N$ samples into a $Q \times P$ matrix $\mathbf{D}=$ $\left[\boldsymbol{D}_{0}, \cdots, \boldsymbol{D}_{P-1}\right]$, where $\boldsymbol{D}_{l}=[\tilde{d}(l), \tilde{d}(l+P), \cdots, \tilde{d}(l+(Q-$ 1) $P)]^{T}$. We have that

$$
\boldsymbol{D}_{l}=\mathbf{G} \tilde{\boldsymbol{d}}_{l}+\boldsymbol{v}_{l}, l=0, \cdots, P-1
$$

where $\tilde{\boldsymbol{d}}_{l}=\left[\tilde{d}^{(1,0)}(l) \tilde{d}^{(1,1)}(l) \cdots \tilde{d}^{(M, V-1)}(l)\right]^{T}, \mathbf{G}=\left[\boldsymbol{G}_{1,0}\right.$ $\left.\boldsymbol{G}_{1,1} \cdots \boldsymbol{G}_{1, V-1} \boldsymbol{G}_{2,0} \cdots \boldsymbol{G}_{M, V-1}\right]$ and $\boldsymbol{G}_{i, v}=\left[1 e^{j \theta_{i, v} P} \ldots\right.$ $\left.e^{j \theta_{i, v}(Q-1) P}\right]^{T}$.

Since $\left|\omega_{i}\right|$ is assumed to be less than 0.5 , the $\theta_{i, v}$ 's are distinct from each other. Moreover, the training OFDM block contains null subcarriers since $Q>M V$. Thus, CFO estimation can be carried out using a signal subspace decomposition approach. The dimension of the null subspace is dictated by the number of null subcarriers. CFO estimation for single user OFDM using null subcarriers was studied in e.g. [8] and references therein. Thus, to estimate the multiple CFOs, subspace based methods (MUSIC and ESPRIT) are known to provide low complexity algorithms, compared to the ML method. Here we adapt one of the modified versions of ESPRIT, namely forward-backward smoothing ESPRIT (FBS-ESPRIT) [9] to our CFO estimation problem. The $\theta_{i, v} \mathrm{~s}$ are estimated using the following steps:

Step 1) Arrange the received signal $\tilde{\boldsymbol{d}}$ into matrix $\mathbf{D}$. The covariance matrix $\mathbf{R}_{d d}=\mathrm{E}\left\{\boldsymbol{D}_{l} \boldsymbol{D}_{l}^{H}\right\}$ of $\boldsymbol{D}_{l}$ is estimated by using forward-backward smoothing as $\widehat{\mathbf{R}}_{d d}=\left(\tilde{\mathbf{R}}_{d d}+\mathbf{J} \tilde{\mathbf{R}}_{d d}^{T} \mathbf{J}\right)$, where $\tilde{\mathbf{R}}_{d d}=\frac{1}{P} \mathbf{D D}^{H}$ and $\mathbf{J}$ is the exchange matrix with 1's on the anti-diagonal and 0 's elsewhere.

Step 2) Computing singular value decomposition (SVD) of $\widehat{\mathbf{R}}_{d d}$, arrange the eigenvectors of $\widehat{\mathbf{R}}_{d d}$ that are associated to the $M V$ largest eigenvalues $\lambda_{1} \geq \lambda_{2} \geq \cdots \geq \lambda_{M V}$ into a $Q \times M V$ matrix $\mathbf{U}_{s}$. Let $\mathbf{U}_{s 1}$ denote the first $(Q-1)$ rows of $\mathbf{U}_{s}$ and
$\mathbf{U}_{s 2}$ denote the last $(Q-1)$ rows of $\mathbf{U}_{s}, \theta_{i, v}$ are estimated as

$$
\hat{\theta}_{i, v}=\frac{1}{P} \arg \left(\beta_{k}\right), \quad k=v+(i-1) V
$$

where $\left\{\beta_{k}\right\}_{k=0}^{M V-1}$ are the eigenvalues of $\boldsymbol{\Xi}=\left(\mathbf{U}_{s 1}^{H} \mathbf{U}_{s 1}\right)^{-1}$ $\mathbf{U}_{s 1}^{H} \mathbf{U}_{s 2}$.

After getting the $\hat{\theta}_{i, v}$ 's, we adopt, for simplicity, equal gain combining (EGC) to compute the CFO estimate for each relay node. Thus, the proposed EGC-FBS-ESPRIT-based CFO estimator, which will be referred to as EFCE, is given by

$$
\hat{\omega}_{i}=\frac{1}{V} \sum_{v=0}^{V-1}\left(\frac{N \hat{\theta}_{i, v}}{2 \pi}-v-\kappa_{i} V\right), \quad i=1, \cdots, M
$$

Another subspace-based method (Spectral MUSIC) has been applied to the interleaved OFDMA uplink system in [7]. However, it is worth pointing out that the interleaved subcarriers allocation is a special case $(V=1)$ of the proposed pilot structure. We will see later that $V=1$ is not a good choice. Moreover, the timing offset of the first arrival signal was assumed to be perfectly known in [7]. Such an assumption may not hold true in practice.

\section{SIMULATION RESULTS}

\subsection{Simulation setup}

Consider a cooperative system with two relay nodes, i.e. $M=2$. The total number of subcarriers of each OFDM block is $N=512$. The training sequences of the relay nodes are uncorrelated and have the same power $\sigma_{t s}^{2}$. The $\mathrm{CP}$ and PP of the training sequences are selected to have lengths equal to 64 and 48 , respectively. In order to satisfy the condition that $\max \left\{\left|\tau_{i}-\tau_{j}\right|\right\}<\min \left\{N_{d l}, N_{p p}\right\}$, we assume that the signal of relay node $R_{1}$ arrives at the destination node first in our simulation setup, and the differential propagation delay $\tau_{2}-\tau_{1}$ is uniformly distributed within the interval $[0,48]$. The tile subchannels allocated to the two relay nodes during the training period are distinct. To make sure $Q$ is larger than $M V$, we select $Q=2^{\left\lfloor\log _{2} M V\right\rfloor+1}$, where $\lfloor a\rfloor$ rounds $a$ to the nearest integer smaller than or equal to $a$. Hence, the number of null subcarriers is $256,256,128,256$ and 192 when $V=1,2,3,4,5$, respectively.

In the simulation, we assume that the CIR length is $L=16$, and the channel taps $h_{i}(l)$ are uncorrelated zero-mean Gaussian random variables with exponential power delay profile $\mathrm{E}\left\{\left|h_{i}(l)\right|^{2}\right\}_{l=0}^{L-1}=$ $C \exp (-0.2 l)$, where $C$ is a scalar factor that ensures that the total energy of the channel taps is normalized to unity. Correspondingly, the SNR of each relay node is equal to $\sigma_{t s}^{2} /\left(N \sigma_{v}^{2}\right)$. The channels for different nodes are assumed uncorrelated.

To quantify the degradation of the BER due to residual timing and CFO, which are equal to the offset estimates fed back by the destination node and the actual offsets, information-bearing blocks are transmitted using a cooperative Alamouti space-time encoding and maximum likelihood detection assuming a perfect channel state information is performed.

\subsection{Performance analysis}

In this section, we compare the estimation performance of the proposed algorithms and the conventional SAGE algorithm in [6]. The SAGE estimation results shown are obtained at the 5th iteration.

The left subfigure of Figure 4 shows the timing estimation performance of the proposed WSCTE with different tile sizes and that of the SAGE algorithm. The estimation performance is measured in terms of averaged (over the channel and relay nodes) standard deviation (STD) of the timing errors. We see that the WSCTE with tile size $V=3$ and $V=5$ outperforms SAGE at lower SNR and the opposite is true at high SNR. It can also be seen that WSCTE performance is dominated by multinode interference since it is not very 

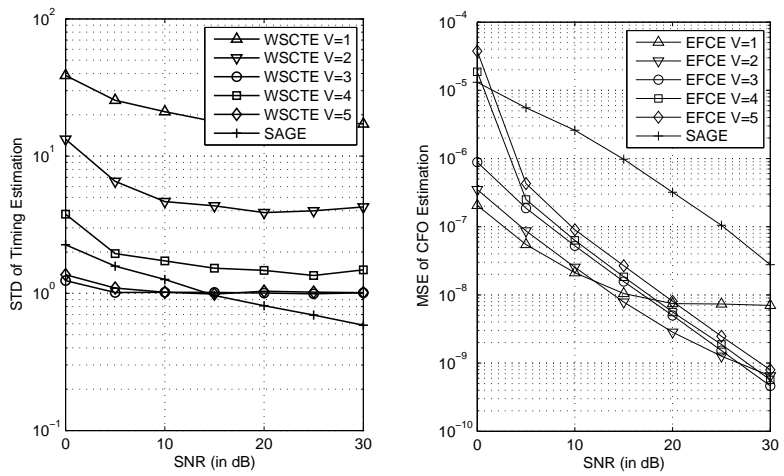

Fig. 4. Estimation performance versus SNR

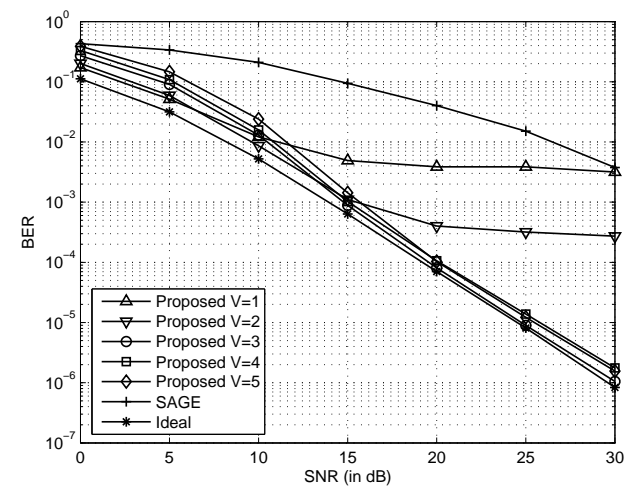

Fig. 5. BER performance versus SNR for uncoded QPSK

sensitive to SNR. The figure shows that the WSCTE performance is improved by increasing the tile size $V$. However, the improvement shrinks for large tile sizes. The tile size $V=3$ seems to provide sufficient accuracy.

The right subfigure of Figure 4 compares the CFO estimation performance of the EFCE with different tile sizes and that of the SAGE algorithm. The performance is measured in terms of averaged (over the channel and relay nodes) MSE. We set the frequency offsets to $\omega_{1}=0.35$ and $\omega_{2}=0.25$. It can be seen that the EFCE algorithm outperforms SAGE significantly. It also shows that the smaller the tile size the better the performance of the EFCE algorithm at low SNR. This is due to the fact that the number of groups, $\mathrm{P}$, or number of null subcarriers decreases when $V$ increases. However, since the timing errors are large for small tile sizes, as shown in the left subfigure, CFO estimation exhibits an error floor for tile sizes $V=1$ and $V=2$ starting from moderate SNRs. Again, tile size $V=3$ seems to be a good choice since the associated error floor does not occurs until the SNR is unrealistically high.

The BER performance of an uncoded QPSK system is shown in Figure 5. A cooperative Alamouti space-time encoding scheme is adopted and the channel state information, which takes into account timing errors, is assumed to be known in the simulation. The length of the CP for the data blocks is set to 20. As expected, SAGE is outperformed by the proposed algorithms. It can be seen that tile size $V=3$ outperforms the other evaluated tile sizes at intermediate and high SNRs, and the corresponding BER performance is close to the ideal case scenario (no synchronization errors).

It is worth pointing out that tile size $V=3$ is optimal for our simulation setup, but a different value may preferred for a system with a different number of subcarriers $N$ and/or relay nodes $M$. In fact, using a statistical analysis of the proposed estimators, the optimal tile size can be predicted analytically. These results, not shown here because of lack of space, will be presented in a longer version of this work

\subsection{Computational Complexity}

We briefly compare the complexities of the proposed synchronization algorithms and SAGE. Considering the number of complex multiplications as a complexity metric, the inversion of an $n \times n$ matrix requires $\mathcal{O}\left(n^{3}\right)$ operations, the SVD of $n \times n$ matrix requires $\mathcal{O}\left(n^{3}\right)$ operations, and the product of an $m \times r$ matrix with a $r \times n$ matrix requires $\mathcal{O}(m r n)$ operations.

Assume that the WSCTE and SAGE algorithms need to search over the same time interval of length, say $K_{t}$, for timing estimation. The complexity of WSCTE is $\mathcal{O}\left(K_{t} M N\right)$, and SAGE needs $\mathcal{O}\left(K_{t} M N L K_{i t}\right)$ operations, where $K_{i t}$ denotes the number of iterations. Regarding CFO estimation, SAGE takes $\mathcal{O}\left(M N L K_{i t} K_{f}\right)$ operations, where $K_{f}>10^{3}$ denotes the number of evaluated CFO offsets in each iteration. Compared to the complicated SAGE algorithm, the proposed EFCE only requires $\mathcal{O}\left(Q^{3}+N Q\right)$ operations.

\section{CONCLUSION}

We have addressed the problem of timing and frequency synchronization in OFDM-based cooperative systems. To avoid multiparameter and multi-dimension search required by the exact ML estimator, we divided the subcarriers of the pilot OFDM block into tile subchannels and performed a correlation-type algorithm for timing synchronization and an ESPRIT-type algorithm, exploiting null subcarriers, for frequency synchronization. By judiciously choosing the tile size, it was shown that the proposed algorithms outperform the existing SAGE algorithm significantly. The proposed algorithms are also computationally much more attractive than SAGE.

\section{REFERENCES}

[1] Y. Mei, Y.B. Hua, A. Swami, and B. Daneshrad, "Combating synchronization errors in cooperative relays," in Proc. IEEE International Conference on Acoustics, Speech, and Signal Processing, 2005, vol. 3, pp. iii/369-iii/372 Vol. 3.

[2] Z. Li and X.G. Xia, "A Simple Alamouti Space-Time Transmission Scheme for Asynchronous Cooperative Systems," Signal Processing Letters, IEEE, vol. 14, no. 11, pp. 804-807, 2007.

[3] F. Ng and X.H. Li, "Cooperative STBC-OFDM Transmissions with Imperfect Synchronization in Time and Frequency," in Conference Record of the Thirty-Ninth Asilomar Conference on Signals, Systems and Computers, 2005, pp. 524-528.

[4] M.-K. Oh, X.L. Ma, G.B. Giannakis, and D.-J. Park, "Cooperative synchronization and channel estimation in wireless sensor networks," in Conference Record of the Thirty-Seventh Asilomar Conference on Signals, Systems and Computers, 2003, vol. 1, pp. 238-242 Vol.1.

[5] M. Morelli, "Timing and frequency synchronization for the uplink of an OFDMA system," IEEE Trans. Commun., vol. 52, no. 2, pp. 296-306, 2004.

[6] J.-H. Lee and S.-C. Kim, "Time and Frequency Synchronization for OFDMA Uplink System using the SAGE Algorithm," IEEE Trans. Wireless Commun., vol. 6, no. 4, pp. 1176-1181, 2007.

[7] Z.R. Cao, U. Tureli, and Y.D. Yao, "Deterministic multiuser carrier-frequency offset estimation for interleaved OFDMA uplink," IEEE Trans. Commun., vol. 52, no. 9, pp. 1585-1594, 2004.

[8] M. Ghogho, A. Swami, and G.B. Giannakis, "Optimized null-subcarrier selection for CFO estimation in OFDM over frequency-selective fading channels," in Proc. IEEE Global Telecommunications Conference, 2001, vol. 1, pp. 202-206 vol.1.

[9] R. Bachl, "The forward-backward averaging technique applied to TLS-ESPRIT processing," IEEE Trans. Signal Process., vol. 43, no. 11, pp. 2691-2699, 1995. 